\title{
Identification of the Zebrafish Ventral Habenula As a Homolog of the Mammalian Lateral Habenula
}

\author{
Ryunosuke Amo, ${ }^{1,2 *}$ Hidenori Aizawa, ${ }^{1,3 *}$ Mikako Takahoko, ${ }^{1}$ Megumi Kobayashi, ${ }^{1}$ Rieko Takahashi, ${ }^{1}$ Tazu Aoki, ${ }^{1}$ \\ and Hitoshi Okamoto ${ }^{1,2,3}$ \\ ${ }^{1}$ Laboratory for Developmental Gene Regulation, RIKEN Brain Science Institute, Wako, Saitama 351-0198, Japan, ${ }^{2}$ Department of Life Science and Medical \\ Bioscience, Graduate School of Advanced Science and Engineering, Waseda University, Shinjuku-ku, Tokyo 162-8480, Japan, and ${ }^{3}$ Core Research for \\ Evolutional Science and Technology, Japan Science and Technology Agency, Chiyoda-ku, Tokyo 102-0075, Japan
}

The mammalian habenula consists of the medial and lateral habenulae. Recent behavioral and electrophysiological studies suggested that the lateral habenula plays a pivotal role in controlling motor and cognitive behaviors by influencing the activity of dopaminergic and serotonergic neurons. Despite the functional significance, manipulating neural activity in this pathway remains difficult because of the absence of a genetically accessible animal model such as zebrafish. To address the level of lateral habenula conservation in zebrafish, we applied the tract-tracing technique to GFP (green fluorescent protein)-expressing transgenic zebrafish to identify habenular neurons that project to the raphe nuclei, a major target of the mammalian lateral habenula. Axonal tracing in live and fixed fish showed projection of zebrafish ventral habenula axons to the ventral part of the median raphe, but not to the interpeduncular nucleus where the dorsal habenula projected. The ventral habenula expressed protocadherin 10a, a specific marker of the rat lateral habenula, whereas the dorsal habenula showed no such expression. Gene expression analyses revealed that the ventromedially positioned ventral habenula in the adult originated from the region of primordium lateral to the dorsal habenula during development. This suggested that zebrafish habenulae emerge during development with mediolateral orientation similar to that of the mammalian medial and lateral habenulae. These findings indicated that the lateral habenular pathways are evolutionarily conserved pathways and might control adaptive behaviors in vertebrates through the regulation of monoaminergic activities.

\section{Introduction}

The mammalian habenula is subdivided into medial and lateral habenulae, each of which project axons to different targets. Specifically, the medial habenula projects to the interpeduncular nucleus (IPN), whereas the lateral habenula projects directly to the median and dorsal raphe, substantia nigra (SN), and ventral tegmental area (VTA) (Herkenham and Nauta, 1979). This organization is also found in lizard, in which the medial and lateral habenulae project to the IPN and the raphe nuclei, respectively (Distel and Ebbesson, 1981).

\footnotetext{
Received July 30, 2009; revised Nov. 23, 2009; accepted Dec. 14, 2009.

This work was supported by grants from RIKEN Brain Science Institute (BSI), Core Research for Evolutional Science and Technology of Japan Science and Technology Agency, and Grant-in-Aid for Scientific Research on Priority Areas KAKENHI20021034I, Scientific Research on Innovative Areas Grant KAKENHI21115521, and Scientific Research (B) Grant KAKENHI19300115 from the Ministry of Education, Culture, Sports, Science and Technology (MEXT) (H.O.), and by Grant-in-Aid for Young Scientists (B) KAKENHI21700370 from MEXT (H.A.). R.A. is a recipient of a fellowship of the Japan Society for the Promotion of Science for Junior Scientists. We thank the members of our laboratories for valuable discussions related to this work; Naoyuki Yamamoto for technical advice on tract tracing; Masakazu Agetsuma, Shusaku Kurisu, Shinya Ohata, Toshiyuki Shiraki, and Hironori Wada for help with plasmid construction; Koich Kawakami for pT2KXIGSin; Stephen Smith for UAS:Syp-GFP; Roger Tsien for mRFP; and Stephen Wilson for the $\mathrm{Tg}$ ( flh:GFP) line. We also thank the RIKEN BSI-Olympus Collaboration Center for confocal microscopy and the RIKEN Research Resource Center for GeneChip analysis.

*R.A. and H.A. contributed equally to this work.

Correspondence should be addressed to Hitoshi Okamoto, Laboratory for Developmental Gene Regulation, RIKEN Brain Science Institute, 2-1 Hirosawa, Wako, Saitama 351-0198, Japan. E-mail: hitoshi@brain.riken.jp.

R. Takahashi's present address: The National Museum of Emerging Science and Innovation, 2-41, Aomi, Koto-ku, Tokyo 135-0064, Japan.

DOI:10.1523/JNEUROSCI.3690-09.2010

Copyright $\odot 2010$ the authors $\quad 0270-6474 / 10 / 301566-09 \$ 15.00 / 0$
}

Among these pathways, it was recently reported that the lateral habenula is activated in response to aversive stimulus as well as outcomes that seem inappropriate for the chosen behaviors, as opposed to the inactivation of the midbrain dopaminergic neurons (Matsumoto and Hikosaka, 2007, 2009). Stimulation of the lateral habenula has an inhibitory effect on both the dopaminergic and serotonergic neurons (Wang and Aghajanian, 1977; Christoph et al., 1986; Matsumoto and Hikosaka, 2007). In addition, destroying the medial and lateral habenular pathways leads to increased monoamine metabolism (Nishikawa and Scatton, 1985; Nishikawa et al., 1986) accompanied by increased locomotion (Lecourtier et al., 2004), reduction of rapid eye movement sleep (Valjakka et al., 1998), and impaired responses to stress (Amat et al., 2001). These studies suggested that the lateral habenula plays a pivotal role in controlling motor and cognitive behaviors by influencing the activity of dopamine and serotonin neurons.

Despite the functional significance, manipulating neural activity in a subset of the habenular pathways remains difficult mainly because of the absence of a model animal in which the neural circuitry is readily amenable to genetic manipulation. Zebrafish are genetically accessible and amenable to imaging neural activities because of their transparency during development. Resolving the neuroanatomy of zebrafish habenulae should therefore inform the functional dissection of neural circuits regulating monoaminergic systems. Fish and amphibian habenulae can be subdivided into dorsal and ventral habenula based on differences 
in cytoarchitecture (Braford and Northcutt, 1983; Kemali and Làzàr, 1985). The zebrafish dorsal habenula projects to the IPN (Aizawa et al., 2005; Gamse et al., 2005) and is thus analogous to the medial habenula of mammals. The dorsal habenula shows conspicuous left-right differences, which recent genetic analyses revealed to emerge in the habenular subnuclei and their projections to the IPN (Aizawa et al., 2007; Carl et al., 2007; Inbal et al., 2007; Kuan et al., 2007; Snelson et al., 2008; Regan et al., 2009; Roussigné et al., 2009). Some neurons in the fish and amphibian habenulae project to the serotonergic neuron-rich raphe nuclei, although studies showing this lacked detailed descriptions of the position of such neurons with respect to the dorsal and ventral organization of the habenula (Kemali and Làzàr, 1985; Yañez and Anadón, 1996; Gugliemotti and Fiorino, 1998; Tomizawa et al., 2001; Aizawa et al., 2005; Bianco et al., 2008). Thus, there is scant experimental evidence of a fish homolog of the lateral habenula, particularly with respect to neural connectivity and molecular characterization of the neurons.

\section{Materials and Methods}

Animals. The study used adult and larval wild-type (RIKEN Wako) and transgenic zebrafish, $\operatorname{Tg}\left(\right.$ brn3a-hsp70:GFP) ${ }^{\text {rw0110b }}$ (Aizawa et al., 2005) and $\operatorname{Tg}(f l h: G F P)^{\mathrm{ul}}$ (Concha et al., 2003), C57BL6J mice (weighing 23-24 g), and Long-Evans rats (weighing 250-300 g).

Tract tracing in fixed fish using carbocyanines. Neuronal cell bodies projecting to the raphe were retrogradely labeled by injecting $1,1^{\prime}$ dioctadecyl-3,3,3',3'-tetramethylindocarbocyanine perchlorate (DiI) (Invitrogen) as described previously (Aizawa et al., 2005). For injections into the median raphe, we injected DiI into the median raphe of adult $\operatorname{Tg}(b r n 3 a-h s p 70: G F P)$, which expressed green fluorescent protein (GFP) in the habenulo-interpeduncular pathway (see Fig. $1 A$ ). Removing the inferior lobe of the hypothalamus of adult $\operatorname{Tg}($ brn3a-hsp 70:GFP) with forceps exposed the ventral aspect of the GFP-expressing IPN and enabled us to identify the median raphe just caudal to the IPN. A crystal of DiI was then inserted with a fine glass needle into the median raphe from the ventral side using the GFP fluorescence in the IPN as a guide.

Tract tracing in live fish with axonal tracers. Fish were anesthetized by immersion in water containing $150 \mathrm{mg} / \mathrm{L}$ tricaine and positioned in a physical restraint device. Anesthetic was also perfused through the gills during surgery to keep fish immobile and to enable artificial respiration. A dorsal portion of the cranium was removed. The tip of the glass micropipette filled with Neurobiotin ( $2 \%$; Vector Laboratories) in $0.5 \mathrm{M}$ $\mathrm{KCl}$ or biotinylated dextran amine, molecular weight 3000 (BDA-3000) (10\%; Invitrogen) in $0.05 \mathrm{M}$ phosphate buffer was stereotaxically positioned in the ventral habenula or the median raphe using stereotaxic micromanipulator (SR-6M and SM-15; Narishige). Coordinates for the ventral habenula were $0-150 \mu \mathrm{m}$ posterior to the anterior margin of the tectum, $10-30 \mu \mathrm{m}$ lateral to the midline, and $150-250 \mu \mathrm{m}$ in depth from the surface of the brain. Coordinates for the median raphe were 1100 $1400 \mu \mathrm{m}$ posterior to the anterior margin of the tectum, $0 \mu \mathrm{m}$ lateral to the midline, and 900-1300 $\mu \mathrm{m}$ in depth from the surface of the brain. The dye was injected iontophoretically by applying a positive current of 5 $\mu \mathrm{A}$ for $50-120 \mathrm{~s}(1 \mathrm{~s}$ on/1 s off) through the stimulus isolation unit (Isoflex; A.M.P.I.) connected to the electric stimulator (SEN-8203; Nihon Kohden). After removal of the electrode, the fish were allowed to recover in water without anesthetic. Animals were maintained postoperatively in fish Ringer's solution (Sajovic and Levinthal, 1982) for 20-30 min (Neurobiotin) or 6-8 h (BDA-3000), and then deeply anesthetized and decapitated. The brains were immersed in $4 \%$ paraformaldehyde (PFA) in PBS at $4^{\circ} \mathrm{C}$ for $16 \mathrm{~h}$. Sections (50-80 $\mu \mathrm{m}$ thickness) were cut using a vibrating microtome and stained with the avidin-biotin peroxidase complex kit (Vector Laboratories), using streptavidin-Alexa Fluor 488 or 546 (Invitrogen) as fluorophore labels.

Plasmids. Plasmid DNAs for electroporation were constructed as follows. pCS2:gal4-VP16 was obtained by subcloning a XbaI-XbaI fragment of gal4-VP16 that was PCR-amplified from pBGal4VP16 (Köster and Fraser, 2001) into an XbaI site of the pCS2 vector (Rupp et al., 1994;
Turner and Weintraub, 1994). For $p C S 2: l y n-G F P$, we first obtained pCS2:AcGFP by subcloning an XbaI-SpeI fragment of AcGFP that was PCR-amplified from $p A c G F P$-Golgi (Clontech) into the XbaI-SpeI sites of pCS2. Synthesized oligonucleotides for the Kosak sequence followed by the N-terminal 10 aa of murine $L y n$ were then subcloned into the BamHI-EcoRI sites of $p C S 2$ :AcGFP to generate $p C S 2: l y n-G F P$. For $p C S 2$ : lyn-RFP, a celsr2-venus fragment of pCS2:lyn-celsr2-venus (CarreiraBarbosa et al., 2009) was replaced with a PCR-amplified EcoRI-XbaI fragment of monomeric RFP (the monomeric RFP plasmid was kindly provided by Roger Tsien, University of California at San Diego, La Jolla, CA). For Tol2-UAS:lyn-RFP, a BamHI-NotI fragment of lyn-RFP was excised from $p C S 2: l y n-R F P$, blunt-ended, and then subcloned into a vector generated by replacing an ApaI-BglII fragment of pT2KXIGAin (Kawakami et al., 2004) with a ClaI-ClaI fragment of UAS, which was PCR-amplified from $p B U A S E 1 B G F P$ (Köster and Fraser, 2001).

Electroporation of adult zebrafish brain. pCS2:lyn-GFP alone or pCS2: gal4-VP16 combined with both Tol2-UAS:lyn-RFP and UAS:syp-GFP (Meyer and Smith, 2006) were transfected into the ventral habenular neurons of adult fish by electroporation as previously reported (Hendricks and Jesuthasan, 2007b). Adult fish were prepared as described for tract tracing in live fish, and a small hole was made using a fine blade in the skull covering the habenulae. A pair of custom-made parallel tungsten electrodes ( $0.1 \mathrm{~mm}$ in diameter; Nisshin EM), spaced $0.5 \mathrm{~mm}$ apart, were inserted so that the left and right habenulae were positioned between them. Plasmid DNA ( $1 \mu \mathrm{g} / \mu \mathrm{l}$ of each) was pressure-injected into the habenula or ventricle using fine-glass micropipettes, followed by one to five $30 \mathrm{~V}$ pulses, each lasting $1 \mathrm{~ms}$, delivered with a Grass SD9 electric stimulator. Animals were maintained postoperatively in fish Ringer's solution for $2-5 \mathrm{~d}$.

GeneChip analysis. The whole brain or the epithalamus, which includes the habenulae and epiphysis of adult $\operatorname{Tg}(b r n 3 a-h s p 70: G F P)$ zebrafish, was dissected manually under the guidance of GFP fluorescence, and total RNA was extracted using the RNAeasy kit (QIAGEN). Synthesis of cRNA probes (One-Cycle Target Labeling kit; Affymetrix), hybridization with GeneChip for zebrafish (14,900 transcripts; Affymetrix), and detection of the fluorescent signals were conducted according to the manufacturer's instructions. The expression levels of all transcripts were compared between epithalamus and whole brain, and genes with specific expression in the epithalamus were identified based on the ratios of the mRNA expression levels in the epithalamus to those in the whole brain (epithalamus/whole-brain ratios) by using the Genescr program as described previously (Zirlinger et al., 2001). We excluded the transcripts whose expressions were previously reported in the zebrafish habenula or were supposed to be expressed in the photosensitive epiphysis because of the possible involvement in the photosensory transduction [e.g., extraocular rhodopsin (Mano et al., 1999)], and finally selected 23 transcripts as candidates that showed more than twofold increase in the epithalamus/ whole-brain ratios. Then, we conducted in situ hybridization screening for these genes in $4 \mathrm{~d}$ postfertilization (dpf) zebrafish and identified three genes that were expressed specifically in the habenulae.

In situ hybridization. In situ hybridization was performed on larval zebrafish and sections of adult brain and other tissues, as described previously (Westerfield, 1995). cRNA probes for adenylate cyclase activating polypeptide 1a (adcyap1a) (Fradinger and Sherwood, 2000), diamine oxidase (Sundström et al., 2008), hypothetical protein LOC335834, protocadherin 10a (pcdh10a) (Woods et al., 2005), pcdh10b (Murakami et al., 2006), and POU domain, class 4, transcription factor 1 (brn3a) (Aizawa et al., 2005) were used for the hybridization reaction. For double hybridizations, a digoxigenin-labeled probe for dao and fluorescein-labeled probe for $p c d h 10 a$ were visualized with the TSA Plus System (PerkinElmer) and FastRed (Roche), respectively.

The cDNA templates for the probes except for brn3a were obtained commercially (B-Bridge International), and the full-length cDNAs were sequenced. The following DNA sequences were used as templates for synthesizing the cRNA probes: IMAGE:5081091, which harbors nucleotides 253-1294 of the adcyapla gene (GenBank accession number NM_152885); IMAGE:3733638, nucleotides 414-1442 of dao (NM_ 001077598); UCDMp611E1817Q, nucleotides 1-655 of hypothetical protein LOC335834 (NM_001080986); IMAGE:6969209, nucleotides 
1-3290 of pcdh10a (NM_212571); and IMAGE:4831442, nucleotides 1435-4277 of $p c d h 10 b$ (NM_182885). For brn3a, we used the full length of the brn3a cDNA as a template for synthesis of cRNA probe (Aizawa et al., 2005).

To detect mRNA in the adult mouse and rat tissues, the animals were deeply anesthetized by intraperitoneal administration of $25 \%$ urethane, and then perfused with $4 \%$ PFA in PBS. Sections ( $80 \mu \mathrm{m}$ thickness) were cut using a vibrating microtome and processed as described for the zebrafish sections. For probe synthesis, the fragment containing nucleotides 2087-3005 of murine Pcdh10 mRNA (NM_001098170; 63.6 and 62.5\% amino acid identity to zebrafish Pcdh10a and Pcdh10b, respectively); nucleotides 441-1593 of rat Dao mRNA (NM_022935; 54.4\% amino acid identity to zebrafish Dao); nucleotides 1827-2451 of murine Brn3a (NM_011143; 78.3\% amino acid identity to zebrafish Brn3a) (Allen Institute for Brain Science, 2009); and nucleotides 538-1087 of rat histamine N-methyltransferase (NM_031044) were PCR-amplified using rat (Dao and histamine N-methyltransferase) or mouse (Pcdh10) genomic DNA as the templates. The amplified fragments were subcloned into the pCRII-TOPO or pCR-BluntII-TOPO vector (Invitrogen), and the recombinant plasmids were used as the templates to synthesize the antisense cRNA probes.

Some sections were further processed for anti-GFP or anti-HuC/D staining.

Immunohistochemistry. An anti-serotonin rabbit polyclonal antibody (1:250-1:3000 dilution; ImmunoStar), anti-GFP rabbit polyclonal antibody (1:250; Santa Cruz Biotechnology), anti-DsRed rabbit polyclonal antibody (1:1000; Clontech), anti-GFP chicken polyclonal antibody (1: 3000; Aves Laboratories), and anti-HuC/D mouse monoclonal antibody (1:50; Abcam) were used as primary antibodies. In the case of $\mathrm{HuC} / \mathrm{D}$ staining, some sections were immersed in $10 \mathrm{~mm}$ sodium citrate buffer, $\mathrm{pH} 6.0$, and heated at $80^{\circ} \mathrm{C}$ for $1 \mathrm{~h}$ before reaction with the primary antibody, to recover antigenicity. Signals were visualized with the antirabbit, anti-chicken, or anti-mouse IgG conjugated with Alexa Fluor 488 , 546,633 , or 647 (1:500; Invitrogen). Some sections were counterstained with Neurotrace red fluorescent Nissl (Invitrogen), 4' ,6'-diamidino-2phenylindole (DAPI) (Invitrogen), or TO-PRO-3 (Invitrogen).

\section{Results \\ The zebrafish ventral habenula projects to the median raphe, but not to the IPN}

The median raphe is a major target of efferent projections from the mammalian lateral habenula (Herkenham and Nauta, 1979), and an identical serotonergic neuron-rich structure was observed at the midline of the zebrafish hindbrain just caudal of the IPN (Fig. $1 B$ ). We predicted that a structure corresponding to the lateral habenula of mammals would contain neurons for projection to the median raphe. The lipophilic dye DiI was injected into the entire region of the median raphe to identify such neurons by retrogradely labeling the habenular cell bodies (Fig. 1C). The raphe was recognized as a ventral hindbrain structure just caudal to the IPN in $\operatorname{Tg}(b r n 3 a-h s p 70: G F P)$ transgenic zebrafish in which the habenulo-interpeduncular pathway expressed GFP (Aizawa et al., 2005). Thus, the retrogradely labeled cell bodies were distributed in the ventral region of the habenula, which was located ventromedially to the GFP-expressing medial subnucleus (Fig. $1 D)$. Tracing in live fish using Neurobiotin revealed that the neurons projecting to the median raphe were distributed ventrolaterally and rostrally, although always remaining within the GFP-negative ventral region of the habenula (Fig. 1E,F). Efferent projection fibers from the ventral region of the habenula were observed in the outer sheath of the fasciculus retroflexus (Fig. 1G, arrows), whereas the GFP-positive efferent projection from the dorsal region appeared in the core of the fasciculus (Fig. 1G, arrowhead). This orientation of the two different habenular efferents in the zebrafish fasciculus retroflexus is similar to that in the medial and lateral habenular efferents in reptiles and mam- mals (Herkenham and Nauta, 1979; Distel and Ebbesson, 1981). This finding further strengthens the demonstrated homology of these pathways across the species. The axons from the ventral region of the habenula circumscribed the IPN and ran caudally to the median raphe (Fig. $1 H$, arrows).

Mammalian medial and lateral habenulae could be identified as the paired epithalamic structures specifically expressing the POU domain transcription factor Brn3a (Fig. 1I) (He et al., 1989). The zebrafish habenula, which specifically expresses brn3a, was subdivided into the dorsal and ventral regions. The dorsal region of the zebrafish habenula showed left-right asymmetry and could be subdivided into the GFP-negative lateral subnucleus and the GFP-positive medial subnucleus in $\operatorname{Tg}(b r n 3 a-$ hsp70:GFP) fish (Fig. 1J) (Aizawa et al., 2005). These two subnuclei in the dorsal region were collectively termed the dorsal habenula and showed stronger expression of the pan-neuronal marker HuC/D (Fig. $1 \mathrm{~K}$ ). The zebrafish dorsal habenula should be structurally homologous to the mammalian medial habenula, since the efferent projections from these regions are mostly restricted to the IPN (Aizawa et al., 2005; Gamse et al., 2005). However, the ventral region was identified by the brn3a-positive/ GFP-negative area and was positioned ventromedially to the dorsal habenula (Fig. 1J). This symmetric region expressed lower levels of $\mathrm{HuC} / \mathrm{D}$ proteins than the dorsal habenula (Fig. $1 \mathrm{~K}$ ). Since the neurons retrogradely labeled from the median raphe were localized in this region, termed the ventral habenula (Fig. $1 L$ ), it seems the most likely candidate site for the fish homolog of the mammalian lateral habenula.

The distinct projection patterns from the dorsal and ventral habenulae were substantiated by anterograde tracing experiments using Neurobiotin injected into either the ventral (Fig. $2 A-D$ ) or dorsal habenula (supplemental Fig. 1, available at www.jneurosci.org as supplemental material). The axons from the ventral habenula passed laterally through the IPN (Fig. $2 B$, arrows) and projected to the ventral part of the median raphe (Fig. $2 C$, arrow; $D$, arrowheads). For a more detailed view of the synaptic terminal distribution, focal electroporation was used to express both membrane-tethered red fluorescent protein (RFP) (Lyn-RFP) and a synaptophysin-GFP (Syp-GFP) fusion protein in the ventral habenular neurons, with signal observed at whole axons and presynaptic terminals, respectively (Fig. 2E-H) (Meyer and Smith, 2006). A small number of ventral habenular neurons expressing these fluorescent proteins consistently projected to the ventral part of the median raphe (Fig. $2 G$, arrow), where punctate expression of Syp-GFP was localized in the axonal varicosities (Fig. $2 \mathrm{H}$, arrowheads). This strongly implicated the puncta as en passant presynaptic terminals. The present analysis revealed no terminal boutons of the ventral habenular axons in direct contact with the serotonin-positive neuronal cell bodies in the raphe $(n=5)$ (Fig. $2 I-L)$. Conversely, axons from the dorsal habenula projected exclusively to the entire IPN, but not to more caudal structures including the median raphe (Aizawa et al., 2005; Gamse et al., 2005) (supplemental Fig. 1, available at www.jneurosci.org as supplemental material).

These results indicated that the zebrafish habenula could be subdivided into the asymmetric dorsal and symmetric ventral habenulae, which projected to the IPN and the ventral part of the median raphe, respectively (Fig. $2 M, N$ ).

\section{The zebrafish ventral habenula shares molecular} characteristics with the mammalian lateral habenula

Homologous structures in the brain of zebrafish can be defined based on similarities among other vertebrates in neural connec- 
A
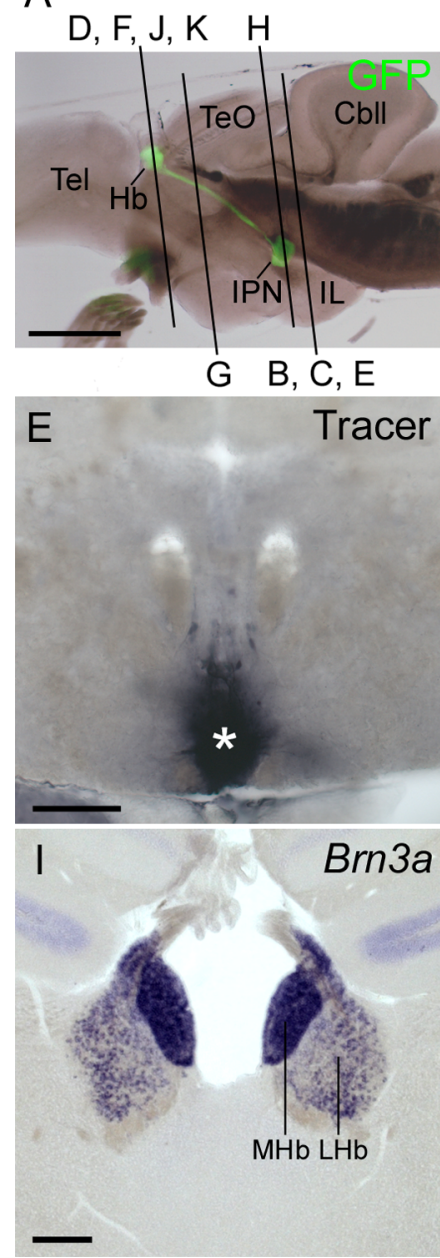
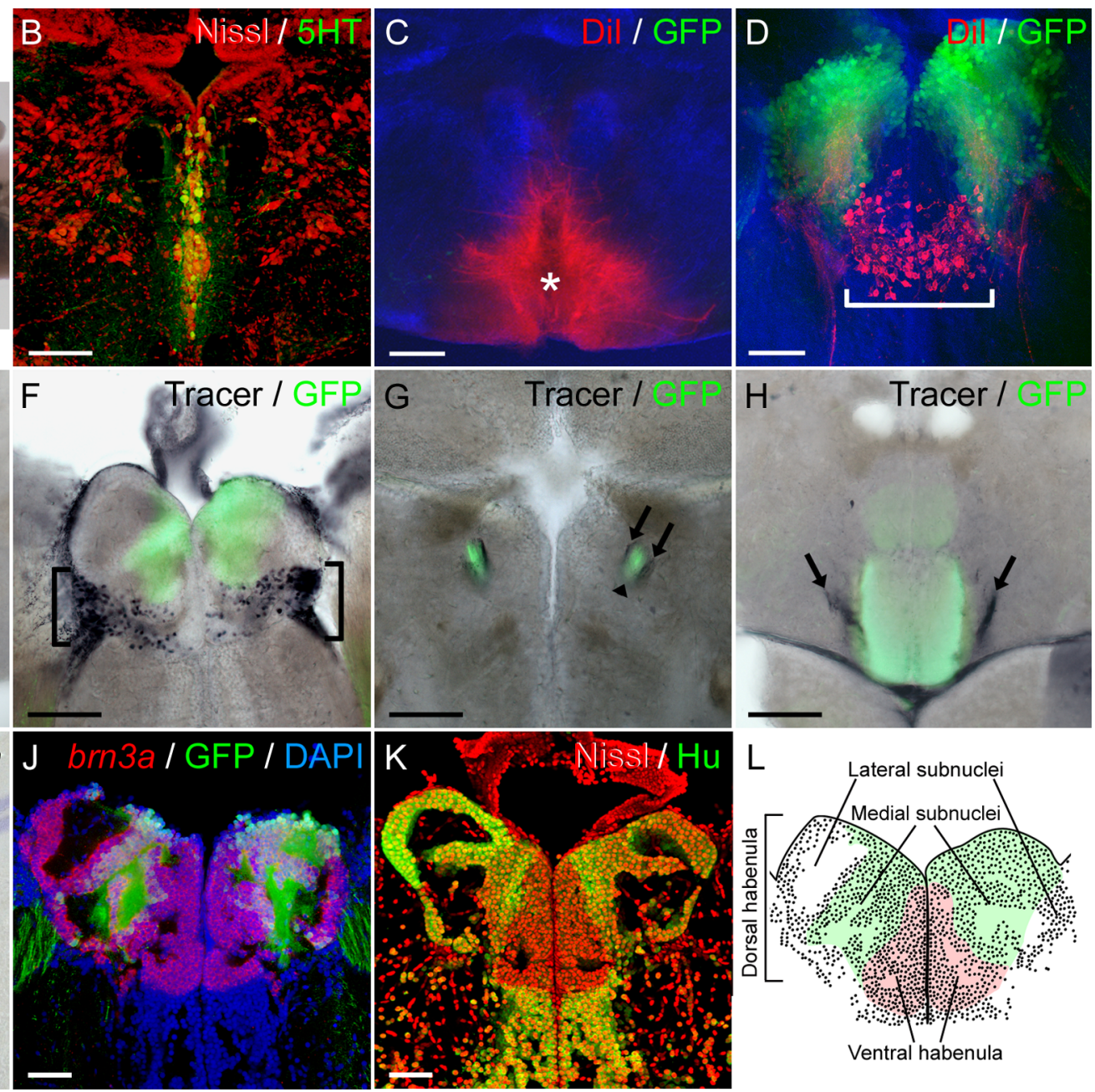

Figure 1. Neurons that project to the median raphe are distributed specifically in the ventral habenula. $A$, Sagittal section of an adult $\operatorname{Tg}(b r n 3 a-h s p 70: G F P)$ showing the habenulo-interpeduncular projection (green). The position of the coronal sections used in the following panels are indicated by oblique vertical bars. $B$, Coronal section of an adult wild-type brain showing the distributions of serotonin-positive neurons in the median raphe (green). $\boldsymbol{C}, \boldsymbol{D}$, Retrograde labeling of neurons in the ventral habenula that project to the median raphe. Coronal sections of an adult $\mathrm{Tg}$ (brn3a-hsp70: GFP) zebrafish showing the Dil injection site in the median raphe ( $\boldsymbol{C}$, asterisk) and the retrogradely labeled cell bodies in the ventral habenula ( $\boldsymbol{D}$, red, bracket). The GFP signal (green) marks the medial subnucleus of the dorsal habenula that projects to the IPN. $\mathbf{E}-\boldsymbol{H}$, Retrograde labeling of neurons in the ventral habenula that project to the median raphe (black). Coronal sections of an adult $\operatorname{Tg}($ brn3a-hsp70:GFP) zebrafish showing the Neurobiotin injection site ( $\boldsymbol{E}$, asterisk), the distribution of retrogradely labeled neurons in the ventral habenula $(\boldsymbol{F}$, brackets), sections of the fasciculi retroflexus ( $\boldsymbol{G}$, the outer sheath indicated by arrows and the core indicated by arrowhead), and the labeled fibers that pass laterally through the GFP-positive IPN ( $\boldsymbol{H}$, arrows). $\boldsymbol{I}, \boldsymbol{J}$, , Oronal sections of an adult mouse brain $(I)$ and $\operatorname{Tg}(b r n 3 a-h s p 70: G F P)$ zebrafish $(J)$ showing murine Brn3a mRNA expression in the medial and lateral habenulae $(I)$ and zebrafish brn3a mRNA (red) in the dorsal and ventral habenulae $(\boldsymbol{J}) . \boldsymbol{K}$, Coronal section of an adult zebrafish habenula showing Hu immunoreactivity. $\boldsymbol{L}$, Schematic diagram of a coronal section of zebrafish habenula showing the dorsal (white and green) and the ventral habenulae (red). The dots indicate the regions rich in cell bodies. The remaining gaps are neuropils. Sections were counterstained with fluorescent NissI ( $\boldsymbol{B}, \boldsymbol{K})$ or DAPI $(\boldsymbol{J})$. Cbll, Cerebellum; Hb, habenula; IL, inferior lobe of the hypothalamus; IPN, interpeduncular nucleus; LHb, lateral habenula; MHb, medial habenula; Tel, telencephalon; Te0, optic tectum. Scale bars: $A, 500 \mu \mathrm{m} ; \boldsymbol{B}, \mathbf{C}, E-H, 100 \mu \mathrm{m} ; \mathbf{D}, J, K, 50 \mu \mathrm{m} ; I, 200 \mu \mathrm{m}$.

tivity and neurochemical characteristics. Identifying a marker that easily characterizes such homologous structures would enable the overall anatomy of the specific structure to be visualized. To address this issue, we searched the literature for genes that are expressed in the mammalian lateral habenula, but not in the mammalian medial habenula.

Protocadherin 10 ( $p c d h 10)$ is a homophilic, cell-cell adhesion molecule, with the murine and rat orthologs expressed specifically in the lateral habenula (Fig. 3A) (Hirano et al., 1999). To verify that this gene expression specificity is conserved in the zebrafish lateral habenula homolog, the adult zebrafish brain was examined for expression of the two zebrafish orthologs [pcdh10a and pcdh10b (Murakami et al., 2006)] of mammalian Pcdh10. Both genes were expressed specifically in the ventral habenula, but not in the dorsal habenula of zebrafish (Fig. $3 B$ for $p c d h 10 a$ ) (data not shown for $p c d h 10 b$ ), and the neurons that were retro- gradely labeled from the median raphe expressed $p c d h 10 a$ (Fig. 3C).

Both zebrafish $p c d h 10 a$ and $p c d h 10 b$ were expressed in the habenula and in many other brain regions including thalamic nuclei (Fig. $3 B$, asterisk). These genes are therefore not suitable markers for delineating the overall structure of the ventral habenula. GeneChip analysis was consequently applied to comprehensively search for genes that are specifically expressed in the zebrafish habenula. In addition to genes already reported to be expressed specifically in the zebrafish habenula [e.g., leftover, cpd2 (Gamse et al., 2005), and proxl (Glasgow and Tomarev, 1998; Thisse and Thisse, 2005)], we found novel genes expressed asymmetrically [i.e., hypothetical protein LOC335834 (Fig. 3D) and adenylate cyclase activating polypeptide $1 a$ (Fradinger and Sherwood, 2000) (Fig. 3E)] or symmetrically [diamine oxidase also called as histaminase or amiloride binding protein 1 (Fig. 3F)] 

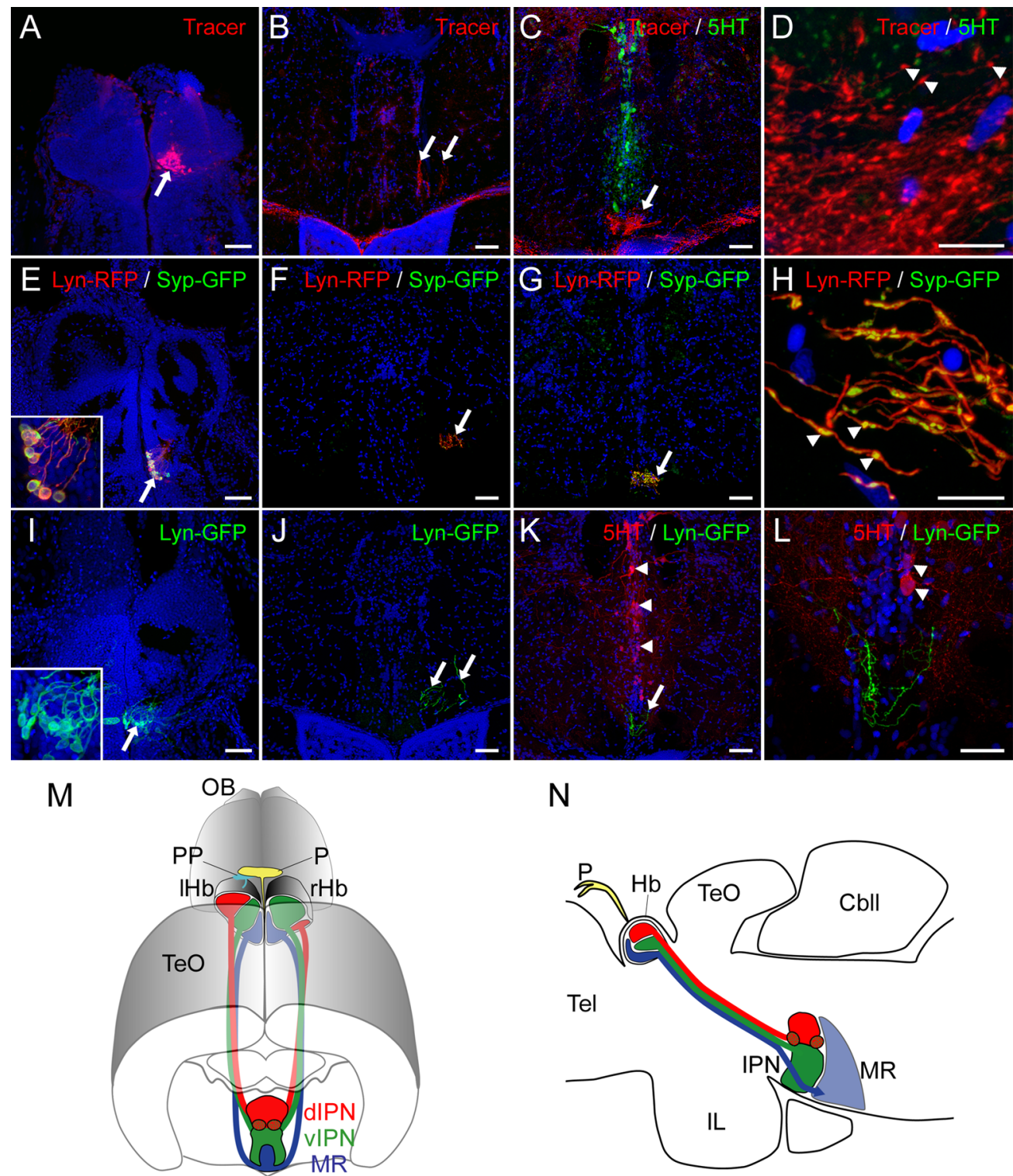

$\mathrm{N}$

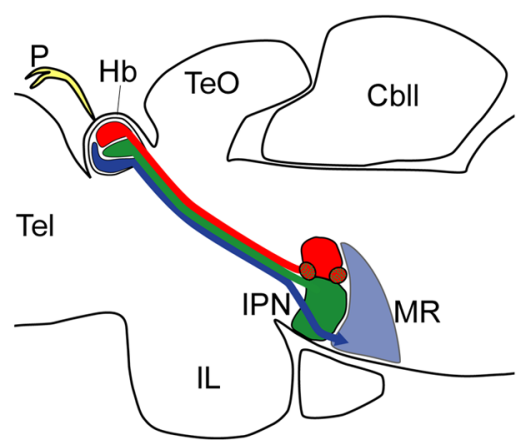

Figure 2. Ventral habenular neurons project to the ventral part of the median raphe. $\boldsymbol{A}-\boldsymbol{L}$, Anterograde tract tracing from the ventral habenula to the median raphe using Neurobiotin $(\boldsymbol{A}-\boldsymbol{D}$, red), transfected Lyn-RFP/Syp-GFP ( $\boldsymbol{E}-\boldsymbol{H}$, red and green, respectively), and transfected Lyn-GFP $(\boldsymbol{I}-\boldsymbol{L}$, green). Coronal sections of the adult zebrafish showing the labeled cell bodies $(\boldsymbol{A}, \boldsymbol{E}, \boldsymbol{I}$, arrows), labeled axons that circumscribe the IPN ( $\boldsymbol{B}, \boldsymbol{F}, \boldsymbol{J}$, arrows), the axons terminated in the ventral part of the median raphe $(\boldsymbol{C}, \boldsymbol{G}, \boldsymbol{K}$, arrows), and the axonal terminals $(\boldsymbol{D}, \boldsymbol{H}$, arrowheads) in the median raphe including serotonin-positive neurons ( $\boldsymbol{C}$, green; $\boldsymbol{K}, \boldsymbol{L}$, red). The insets in $\boldsymbol{E}$ and $\boldsymbol{I}$ show higher magnification of the regions indicated by arrows in $\boldsymbol{E}$ and $\boldsymbol{I}$, respectively. $\boldsymbol{D}, \boldsymbol{H}$, and $\boldsymbol{L}$ show higher magnification of the regions indicated by arrows in $\boldsymbol{C}, \boldsymbol{G}$, and $\boldsymbol{K}$, respectively. The arrowheads in $\boldsymbol{K}$ and $\boldsymbol{L}$ indicate the serotonin-positive neurons. Sections were counterstained with DAPI (blue). $M, N$, Schematic diagram showing the dorsal oblique $(\boldsymbol{M})$ and lateral $(\boldsymbol{N})$ views of the axonal projections from the dorsal (red and green) and ventral habenulae (blue) in the adult zebrafish brain. The left and right dorsal habenulae are subdivided into lateral (red) and medial (green) subnuclei and show asymmetry in subnuclear size. $d$, Dorsal; $I$, left; $M R$, median raphe; $O B$, olfactory bulb; $P$, pineal organ; PP, parapineal organ; r, right; $v$, ventral. Scale bars: $A-C, E-G, I-K, 50 \mu \mathrm{m} ; \boldsymbol{D}, \boldsymbol{H}, 10 \mu \mathrm{m} ; \boldsymbol{L}, 25 \mu \mathrm{m}$.

in $4 \mathrm{dpf}$ larval zebrafish habenula. Among them, we identified diamine oxidase (dao) gene as a specific marker for the ventral habenula by the relevant expression analysis in adult zebrafish brain (Fig. 3G). Although mRNA expression of diamine oxidase is not present in the mammalian brain (Burkard et al., 1963) (supplemental Fig. 2, available at www.jneurosci.org as supplemental material), the expression pattern of dao completely overlapped with that of pcdh10a and localized specifically to the zebrafish ventral habenula (Fig. $3 \mathrm{H}, \mathrm{I}$ ). The dao gene expression pattern also facilitated identification of the entire ventral habenula in serial coronal sections (Fig. $3 J-L$ ). The distributions of the dao-expressing cells expanded mediolaterally, mostly in the rostral part (Fig. $3 J$ ), and then gradually relocated to the ventromedial region of the habenula in the caudal part (Fig. $3 K, L$ ). These expression patterns were completely complementary to the dorsal habenula whose ventral margin was demarcated by brn3ahsp70:GFP expression (Fig. 3G).

Dorsoventral organization of the zebrafish habenula results from dynamic morphogenetic changes

Since the ventral habenula is located ventromedially to the dorsal habenula in the adult zebrafish brain, the orientations of these structures appear opposite to that of the mammalian habenula in the mediolateral direction (Fig. 4C-E). Identification of the ventral habenula using the specific marker gene dao enabled examination of ventral habenula development (Fig. 3M-O). Intriguingly, at $5 \mathrm{dpf}$, dao was first expressed in the habenular region lateral to the GFP-positive dorsal habenula primordium 

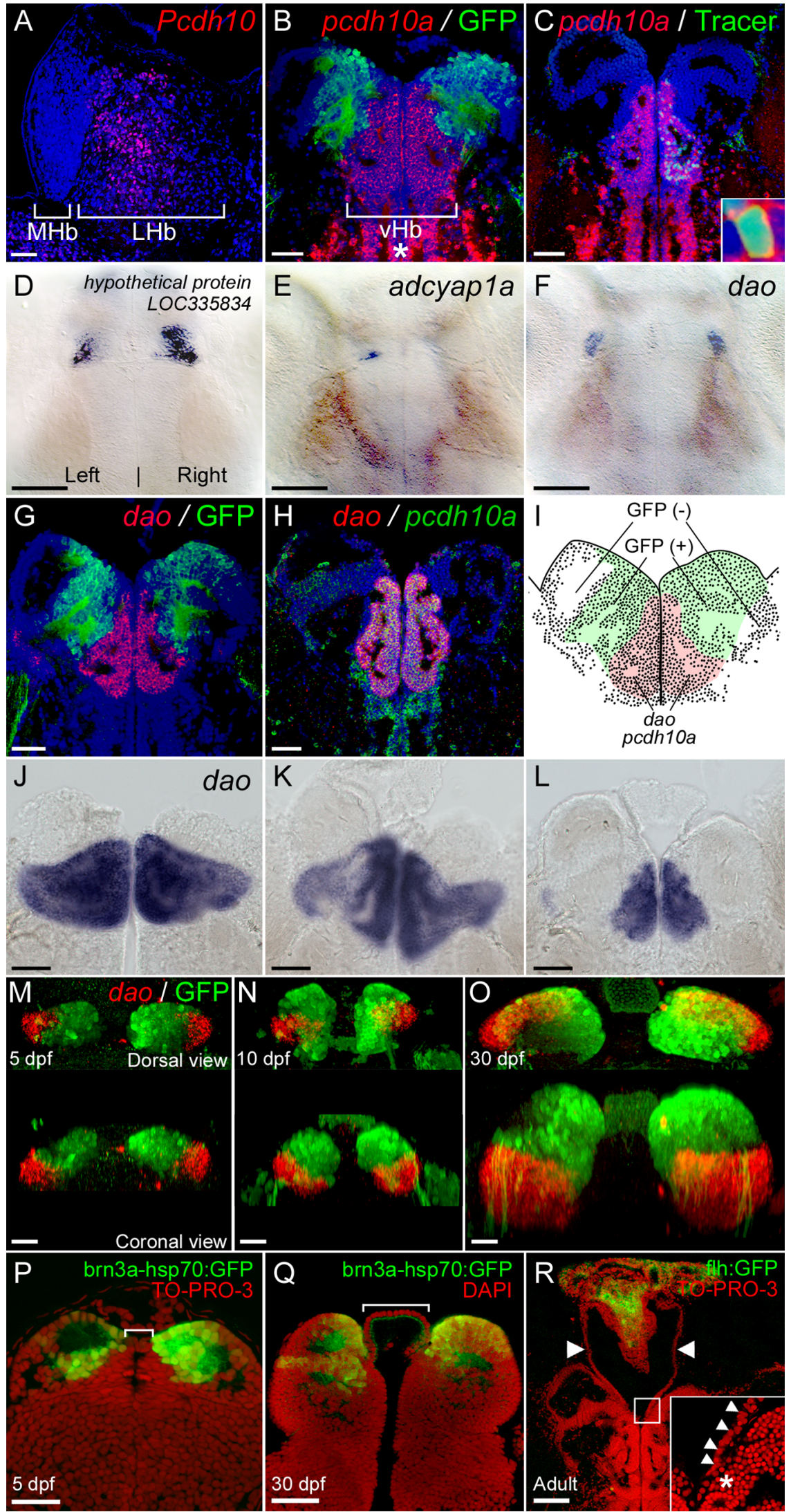

Figure 3. Evolutionary conservation of molecular profiles between the fish ventral habenula and mammalian lateral habenula. $\boldsymbol{A}, \boldsymbol{B}$, Coronal sections of an adult rat $(\boldsymbol{A})$ and an adult $\operatorname{Tg}($ brn3a-hsp 70:GFP) zebrafish (B) showing the expression patterns of rat $P c d h 10$ in the lateral habenula $(\boldsymbol{A}$, red) and zebrafish $p c d h 10 a$ in the ventral habenula $(\boldsymbol{B}$, red). vHb, Ventral habenula. The green coloration in $\boldsymbol{B}$ represents brn3a-hsp70:GFP transgene expression in the medial subnucleus of the dorsal habenula. The sections were stained with anti-HuC/D antibody ( $\boldsymbol{A}$, pseudocolored blue) or DAPI ( $\boldsymbol{B}$, blue). $\boldsymbol{C}$, Retrograde labeling of neurons that project to
(Fig. 3M). The dao-expressing regions gradually migrated ventromedially as development progressed (Fig. $3 N$ ), extending rostrally in the $30 \mathrm{dpf}$ zebrafish brain (Fig. 3O). After these morphogenetic changes, the dao-expressing cell clusters settled at a ventromedial position within the habenula (Fig. $3 G$ ). These observations suggested that the dorsoventral organization of the fish habenula results from dynamic morphogenetic changes (Fig. $4 A-C$ ). If the morphogenetic movements in this region result from the eversion process in which the roofplate of the telencephalon expands laterally (Nieuwenhuys et al., 1998), the contact sites of the roofplates with the habenulae should gradually shift from medial to lateral margins of the habenulae according to the development process. However, this possibility is unlikely, since we observed the diencephalic roof bridging the left and right habenulae in contact with the medial margin of larval (Fig. 3P), juvenile (Fig. 3Q), and adult (Fig. 3R) dorsal habenulae. Therefore,

$\leftarrow$

the median raphe. A coronal section of wild-type zebrafish showing Neurobiotin (green), pcdh10a (red), and nuclear staining (DAPl; blue). The inset shows higher magnification of a retrogradely labeled neuron (green) coexpressing $p c d h 10 a$ (yellow). $\boldsymbol{D}-\boldsymbol{F}$, GeneChip analysis identified genes specifically expressed in the zebrafish habenula. Dorsal views of the $4 \mathrm{dpf}$ wild-type zebrafish brain showing the expression of hypothetical protein LOC335834 (D), adenylate cyclase activating polypeptide $1 a(\boldsymbol{E})$, and diamine oxidase $(\boldsymbol{F}) . \mathbf{G}, \boldsymbol{H}$, Coronal sections of an adult $\mathrm{Tg}$ (brn3a-hsp70:GFP) (G) and wild-type $(\boldsymbol{H})$ zebrafish localizing the expression of dao (red), brn3a-hsp70: GFP ( $G$, green), and pcdh10a ( $\boldsymbol{H}$, green), as well as nuclear staining with DAPI (blue). $I$, Schematic diagram of a coronal section of adult zebrafish habenula, summarizing the expression patterns of those genes expressed specifically in the habenular subregions. $\boldsymbol{J}-\boldsymbol{L}$, Serial coronal sections of the zebrafish wild-type brain at the levels of the rostral ( $)$, middle $(\boldsymbol{K})$, and caudal $(\boldsymbol{L})$ regions of the ventral habenula, showing the expression of dao. $\mathbf{M - 0}$, Development of the ventral habenula, as revealed by dao expression patterns. Dorsal (top panels) and coronal (bottom panels) views of the $\mathrm{Tg}$ (brn3a-hsp70:GFP) zebrafish showing the distributions of dao-expressing cells in the ventral habenula (red) and GFPexpressing cells in the medial subnucleus of the dorsal habenula (green). Developmental stages are shown in the left bottom corner of the top panels. $\boldsymbol{P}-\boldsymbol{R}$, Diencephalic roof remained in contact with the medial margin of the dorsal habenula throughout development. Coronal sections of the $5 \mathrm{dpf}$ (P) and $30 \mathrm{dpf}$ (Q) $\mathrm{Tg}$ (brn3a-hsp70:GFP) and adult $\mathrm{Tg}$ ( flh:GFP) zebrafish $(\boldsymbol{R})$ showing roofplate tela $(\boldsymbol{P}, \mathbf{Q}$, brackets) contacting the GFP-expressing medial subnucleus of the dorsal habenula $(\boldsymbol{P}, \mathbf{Q}$, green), pineal organ ( $\boldsymbol{R}$, green), and saccus dorsalis (presumptive derivative of the embryonic roofplate in the adult brain; $\boldsymbol{R}$, arrowheads). The inset is a higher magnification of the boxed area in $\boldsymbol{R}$ showing the saccus dorsalis (arrowheads) attached to the medial margin of the habenula (asterisk). Sections were counterstained with T0-PRO-3 ( $\boldsymbol{P}, \boldsymbol{R}$, red) or DAPI ( $\boldsymbol{Q}$, red). Scale bars: $\boldsymbol{A}, \boldsymbol{R}, 100 \mu \mathrm{m} ; \boldsymbol{B}-\boldsymbol{H}, \boldsymbol{J}-\boldsymbol{L}, \mathbf{Q}, 50$ $\mu \mathrm{m} ; \boldsymbol{M}-\boldsymbol{P}, 25 \mu \mathrm{m}$. 
morphogenetic movements in the habenular region might be different from those in the fish telencephalon.

\section{Discussion}

This is the first report precisely identifying a nonmammalian homolog of the mammalian lateral habenula. We identified the zebrafish dorsal and ventral habenulae as homologs of the mammalian medial and lateral habenulae, respectively, by analyzing neural connectivity and the molecular characteristics of the habenular neurons.

\section{Evolutionary changes to the mediolateral relationship among corresponding structures of the habenula between mammals and zebrafish}

The lack of a molecular marker that discriminates between the dorsal and ventral habenulae makes it difficult to experimentally identify the region of the fish habenula that is homologous to the mammalian lateral habenula. The present study described specific genetic markers for this structure and used these markers to visualize the entire structure and elucidate the developmental origin of the fish homolog of the mammalian lateral habenula (Fig. $4 A-C$ ). Intriguingly, among vertebrates, fish and primates show completely opposite orientations of the medial and lateral habenular homologs in the mediolateral direction (Fig. 4C-E) (Mikula et al., 2007). In macaque monkeys, the lateral habenula is expanded dorsally, whereas the medial habenula is pushed ventromedially and appears to be residual. This contrasts with the fish habenula organization and suggests that the differential expansion of the medial or lateral habenula in each species reflects the extent of the cortical input through the basal ganglia to the lateral habenula (Yañez and Anadón, 1996).

\section{Conservation of molecular characteristics between the} zebrafish ventral habenula and mammalian lateral habenula The present study revealed that zebrafish ventral and mammalian lateral habenulae both express a lateral habenular marker (pcdh10) and a pan-habenular marker (brn3a) (Aizawa et al., 2007). However, it should be noted that the zebrafish ventral habenula and mammalian lateral habenula might have some differences in addition to the similarities, since diamine oxidase was detected only in zebrafish and not in rat. Histamine is degraded by two pathways: via oxidative deamination by diamine oxidase and via methylation by histamine $N$-methyltransferase (Schayer, 1959). As a consequence of evolution, the inactivating mechanism for histamine has become dependent on the methylation pathway in the mammalian brain, since the mammalian brain expresses histamine $N$-methyltransferase, but not diamine oxidase (Burkard et al., 1963; Almeida and Beaven, 1981; Kitanaka et al., 2002). Although histamine N-methytransferase mRNA expression in the rat habenula was too low to detect by in situ hybridization (data not shown), we cannot exclude the possibility that the histamine N-methyltransferase mRNA is expressed more in the lateral habenula than the medial habenula.

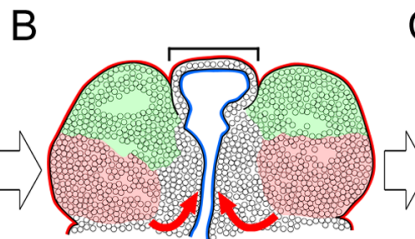

Teleost (Zebrafish, $30 \mathrm{dpf}$ )

E

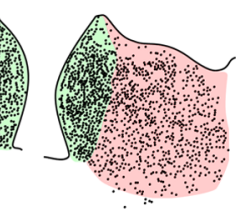

Rodent

(Rat)

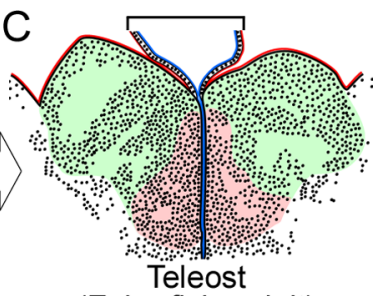

(Zebrafish, adult)

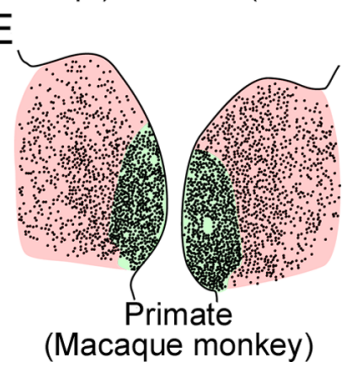

(Macaque monkey)

Figure 4. Medial- and lateral-habenula homologs in vertebrates. Schematic drawings of coronal sections at the level of the habenula, showing regions homologous to the mammalian medial (light green) and lateral habenulae (pink). The circles $(\boldsymbol{A}, \boldsymbol{B})$ and dots $(\boldsymbol{C}-\boldsymbol{E})$ indicate the regions rich in cell bodies. The remaining gaps are neuropils. $\boldsymbol{A}-\boldsymbol{C}$, Schematics with modifications $(\boldsymbol{A})$ and saccus dorsalis $(\boldsymbol{B}, \boldsymbol{C})$, respectively. The red and blue lines indicate the pia mater and ventricular surface, respectively. $\boldsymbol{D}$, Schematic with modification based on Figure $3 A$ showing a coronal section of the rat habenula. $\boldsymbol{E}$, Schematic modified from data

Conservation of neural connectivities between the zebrafish ventral habenula and mammalian lateral habenula

Previous studies demonstrated the mammalian lateral habenula projecting to both the dopaminergic SN/VTA and the serotonergic raphe nuclei. The diencephalic dopaminergic neurons that send the ascending projection to the ventral telencephalon are proposed homologs of the midbrain dopaminergic neurons in mammals (Rink and Wullimann, 2001). However, the present analysis revealed no direct projection from the ventral habenula to the diencephalon, which should include the dopaminergic neurons (Rink and Wullimann, 2001). Hence, the main targets of the efferent projections from the zebrafish dorsal and ventral habenulae seemed to be the IPN and median raphe, respectively. Although we confirmed that the ventral habenula neurons terminated in the ventral region of the median raphe, we did not observe direct contact with serotonergic neuronal cell bodies. Thus, some interneurons may intervene between the habenular termini and the serotonergic neurons. Recent studies in mammals identified the GABAergic pontine rostromedial tegmental nucleus (RMTg) as a relay nucleus connecting the lateral habenula with the dopaminergic SN/VTA (Jhou et al., 2009a,b; Kaufling et al., 2009). Therefore, it is worth examining whether the ventral region of the median raphe may also be involved in regulation of dopamine neurons as the mammalian RMTg.

This study did not examine conservation of the habenular afferents between fish and mammals. The mammalian lateral habenula receives substantial inputs from both the basal ganglia (entopeduncular nucleus, a rodent homolog of primate globus pallidus internal segment, and ventral striatum) and the lateral hypothalamic nucleus, whereas the mammalian medial habenula receives inputs from the limbic structures such as the posterior septum and diagonal band of Broca (Herkenham and Nauta, 1977). A concept of conservation between fish and mammals among the forebrain structures recently emerged (Wullimann and Mueller, 2004). Although previous studies suggested that fish 
habenula receives its inputs from forebrain structures such as the entopeduncular complex, preoptic nucleus, and olfactory bulb, no precise reference has been made to the dorsal and ventral organization of the habenula (Yañez and Anadón, 1996; Hendricks and Jesuthasan, 2007a; Miyasaka et al., 2009). Whether the dorsal and ventral habenulae receive inputs from the telencephalic structures corresponding to the mammalian septum and internal segment of globus pallidus, respectively, remains an intriguing question. Additional investigation into the connectivity of these pathways will elucidate the extent of conservation between habenular afferent circuits in fish and mammals.

Despite the homology demonstrated in efferent projections and gene expression patterns between the fish ventral and mammalian lateral habenula, it remains unknown whether the ventral habenula has the same electrophysiological responses to aversive stimulus and negative reward in fish as in primates (Matsumoto and Hikosaka, 2007, 2009) and whether the axonal terminals of the ventral habenula contact with GABAergic neurons in the raphe as postulated in mammals (Wang and Aghajanian, 1977). Addressing these issues in the future will further clarify the similarities and differences between the regulatory mechanisms of dopaminergic and serotonergic activities in vertebrates.

Finally, despite the emerging physiological evidence that the habenula regulates monoaminergic activities, manipulating neural activity in a subset of these pathways remains difficult mainly because of the absence of the model animal in which the neural circuitry is readily amenable to a genetic approach. Molecular analysis of the habenular pathways conserved in zebrafish is therefore an important step to elucidating the essential role of monoaminergic circuitry in regulating adaptive behaviors in vertebrates.

\section{References}

Aizawa H, Bianco IH, Hamaoka T, Miyashita T, Uemura O, Concha ML, Russell C, Wilson SW, Okamoto H (2005) Laterotopic representation of left-right information onto the dorso-ventral axis of a zebrafish midbrain target nucleus. Curr Biol 15:238-243.

Aizawa H, Goto M, Sato T, Okamoto H (2007) Temporally regulated asymmetric neurogenesis causes left-right difference in the zebrafish habenular structures. Dev Cell 12:87-98.

Allen Institute for Brain Science (2009) Allen mouse brain atlas. Seattle: Allen Institute for Brain Science. Available at http://mouse.brain-map.org.

Almeida AP, Beaven MA (1981) Phylogeny of histamine in vertebrate brain Brain Res 208:244-250.

Amat J, Sparks PD, Matus-Amat P, Griggs J, Watkins LR, Maier SF (2001) The role of the habenular complex in the elevation of dorsal raphe nucleus serotonin and the changes in the behavioral responses produced by uncontrollable stress. Brain Res 917:118-126.

Bianco IH, Carl M, Russell C, Clarke JD, Wilson SW (2008) Brain asymmetry is encoded at the level of axon terminal morphology. Neural Dev 3:9.

Braford MRJ, Northcutt RG (1983) Organization of the diencephalon and pretectum of ray-finned fishes. In: Fish neurobiology. 2. Higher brain areas and functions (Davis RE, Northcutt RG, eds), pp 117-163. Ann Arbor, MI: University of Michigan.

Burkard WP, Gey KF, Pletscher A (1963) Diamine oxidase in the brain of vertebrates. J Neurochem 10:183-186.

Carl M, Bianco IH, Bajoghli B, Aghaallaei N, Czerny T, Wilson SW (2007) Wnt/Axin 1/beta-catenin signaling regulates asymmetric nodal activation, elaboration, and concordance of CNS asymmetries. Neuron 55:393-405.

Carreira-Barbosa F, Kajita M, Kajita M, Morel V, Wada H, Okamoto H, Martinez Arias A, Fujita Y, Wilson SW, Tada M (2009) Flamingo regulates epiboly and convergence/extension movements through cell cohesive and signalling functions during zebrafish gastrulation. Development 136:383-392.

Christoph GR, Leonzio RJ, Wilcox KS (1986) Stimulation of the lateral habenula inhibits dopamine-containing neurons in the substantia nigra and ventral tegmental area of the rat. J Neurosci 6:613-619.

Concha ML, Russell C, Regan JC, Tawk M, Sidi S, Gilmour DT, Kapsimali M,
Sumoy L, Goldstone K, Amaya E, Kimelman D, Nicolson T, Gründer S, Gomperts M, Clarke JD, Wilson SW (2003) Local tissue interactions across the dorsal midline of the forebrain establish CNS laterality. Neuron 39:423-438.

Distel H, Ebbesson SO (1981) Habenular projections in the monitor lizard (Varanus benegalensis). Exp Brain Res 43:324-329.

Fradinger EA, Sherwood NM (2000) Characterization of the gene encoding both growth hormone-releasing hormone (GRF) and pituitary adenylate cyclase-activating polypeptide (PACAP) in the zebrafish. Mol Cell Endocrinol 165:211-219.

Gamse JT, Kuan YS, Macurak M, Brösamle C, Thisse B, Thisse C, Halpern ME (2005) Directional asymmetry of the zebrafish epithalamus guides dorsoventral innervation of the midbrain target. Development 132:48694881.

Glasgow E, Tomarev SI (1998) Restricted expression of the homeobox gene prox 1 in developing zebrafish. Mech Dev 76:175-178.

Gugliemotti V, Fiorino L (1998) Asymmetry in the left and right habenulointerpeduncular tracts in the frog. Brain Res Bull 45:105-110.

Hendricks M, Jesuthasan S (2007a) Asymmetric innervation of the habenula in zebrafish. J Comp Neurol 502:611-619.

Hendricks M, Jesuthasan S (2007b) Electroporation-based methods for in vivo, whole mount and primary culture analysis of zebrafish brain development. Neural Dev 2:6.

Herkenham M, Nauta WJ (1977) Afferent connections of the habenular nuclei in the rat. A horseradish peroxidase study, with a note on the fiber-of-passage problem. J Comp Neurol 173:123-146.

Herkenham M, Nauta WJ (1979) Efferent connections of the habenular nuclei in the rat. J Comp Neurol 187:19-47.

He X, Treacy MN, Simmons DM, Ingraham HA, Swanson LW, Rosenfeld MG (1989) Expression of a large family of POU-domain regulatory genes in mammalian brain development. Nature 340:35-41.

Hirano S, Yan Q, Suzuki ST (1999) Expression of a novel protocadherin, OL-protocadherin, in a subset of functional systems of the developing mouse brain. J Neurosci 19:995-1005.

Inbal A, Kim SH, Shin J, Solnica-Krezel L (2007) Six3 represses nodal activity to establish early brain asymmetry in zebrafish. Neuron 55:407-415.

Jhou TC, Fields HL, Baxter MG, Saper CB, Holland PC (2009a) The rostromedial tegmental nucleus (RMTg), a GABAergic afferent to midbrain dopamine neurons, encodes aversive stimuli and inhibits motor responses. Neuron 61:786-800.

Jhou TC, Geisler S, Marinelli M, Degarmo BA, Zahm DS (2009b) The mesopontine rostromedial tegmental nucleus: a structure targeted by the lateral habenula that projects to the ventral tegmental area of Tsai and substantia nigra compacta. J Comp Neurol 513:566-596.

Kaufling J, Veinante P, Pawlowski SA, Freund-Mercier MJ, Barrot M (2009) Afferents to the GABAergic tail of the ventral tegmental area in the rat. J Comp Neurol 513:597-621.

Kawakami K, Takeda H, Kawakami N, Kobayashi M, Matsuda N, Mishina M (2004) A transposon-mediated gene trap approach identifies developmentally regulated genes in zebrafish. Dev Cell 7:133-144.

Kemali M, Làzàr G (1985) Cobalt injected into the right and left fasciculi retroflexes clarifies the organization of this pathway. J Comp Neurol 233:1-11.

Kitanaka N, Kitanaka J, Oue T, Tada Y, Tanaka T, Takemura M (2002) Genomic structure of the rat and mouse histamine $N$-methyltransferase gene. Jpn J Pharmacol 88:85-92.

Köster RW, Fraser SE (2001) Tracing transgene expression in living zebrafish embryos. Dev Biol 233:329-346.

Kuan YS, Yu HH, Moens CB, Halpern ME (2007) Neuropilin asymmetry mediates a left-right difference in habenular connectivity. Development 134:857-865.

Lecourtier L, Neijt HC, Kelly PH (2004) Habenula lesions cause impaired cognitive performance in rats: implications for schizophrenia. Eur J Neurosci 19:2551-2560.

Mano H, Kojima D, Fukada Y (1999) Exo-rhodopsin: a novel rhodopsin expressed in the zebrafish pineal gland. Brain Res Mol Brain Res 73:110-118.

Matsumoto M, Hikosaka O (2007) Lateral habenula as a source of negative reward signals in dopamine neurons. Nature 447:1111-1115.

Matsumoto M, Hikosaka O (2009) Representation of negative motivational value in the primate lateral habenula. Nat Neurosci 12:77-84.

Meyer MP, Smith SJ (2006) Evidence from in vivo imaging that synaptogen- 
esis guides the growth and branching of axonal arbors by two distinct mechanisms. J Neurosci 26:3604-3614.

Mikula S, Trotts I, Stone JM, Jones EG (2007) Internet-enabled highresolution brain mapping and virtual microscopy. Neuroimage 35:9-15.

Miyasaka N, Morimoto K, Tsubokawa T, Higashijima S, Okamoto H, Yoshihara Y (2009) From the olfactory bulb to higher brain centers: genetic visualization of secondary olfactory pathways in zebrafish. J Neurosci 29: $4756-4767$.

Murakami T, Hijikata T, Matsukawa M, Ishikawa H, Yorifuji H (2006) Zebrafish protocadherin 10 is involved in paraxial mesoderm development and somitogenesis. Dev Dyn 235:506-514.

Nieuwenhuys R, Donkelaar HJ, Nicholson C (1998) In: The central nervous system of vertebrates, pp 166-172. Berlin: Springer-Verlag.

Nishikawa T, Scatton B (1985) Inhibitory influence of GABA on central serotonergic transmission. Involvement of the habenulo-raphe pathways in the GABAergic inhibition of ascending cerebral serotonergic neurons. Brain Res 331:81-90.

Nishikawa T, Fage D, Scatton B (1986) Evidence for, and nature of, the tonic inhibitory influence of habenulointerpeduncular pathways upon cerebral dopaminergic transmission in the rat. Brain Res 373:324-336.

Regan JC, Concha ML, Roussigné M, Russell C, Wilson SW (2009) An Fgf8-dependent bistable cell migratory event establishes CNS asymmetry. Neuron 61:27-34.

Rink E, Wullimann MF (2001) The teleostean (zebrafish) dopaminergic system ascending to the subpallium (striatum) is located in the basal diencephalon (posterior tuberculum). Brain Res 889:316-330.

Roussigné M, Bianco IH, Wilson SW, Blader P (2009) Nodal signalling imposes left-right asymmetry upon neurogenesis in the habenular nuclei. Development 136:1549-1557.

Rupp RA, Snider L, Weintraub H (1994) Xenopus embryos regulate the nuclear localization of XMyoD. Genes Dev 8:1311-1323.

Sajovic P, Levinthal C (1982) Visual cells of zebrafish optic tectum: mapping with small spots. Neuroscience 7:2407-2426.

Schayer RW (1959) Catabolism of physiological quantities of histamine in vivo. Physiol Rev 39:116-126.
Snelson CD, Santhakumar K, Halpern ME, Gamse JT (2008) Tbx2b is required for the development of the parapineal organ. Development 135:1693-1702.

Sundström G, Larsson TA, Larhammar D (2008) Phylogenetic and chromosomal analyses of multiple gene families syntenic with vertebrate Hox clusters. BMC Evol Biol 8:254.

Thisse C, Thisse B (2005) High throughput expression analysis of ZFmodels consortium clones. ZFIN direct data submission. Eugene, OR: Zebrafish Information Network. Available at http://zfin.org.

Tomizawa K, Katayama H, Nakayasu H (2001) A novel monoclonal antibody recognizes a previously unknown subdivision of the habenulointerpeduncular system in zebrafish. Brain Res 901:117-127.

Turner DL, Weintraub H (1994) Expression of achaete-scute homolog 3 in Xenopus embryos converts ectodermal cells to a neural fate. Genes Dev 8:1434-1447.

Valjakka A, Vartiainen J, Tuomisto L, Tuomisto JT, Olkkonen H, Airaksinen MM (1998) The fasciculus retroflexus controls the integrity of REM sleep by supporting the generation of hippocampal theta rhythm and rapid eye movements in rats. Brain Res Bull 47:171-184.

Wang RY, Aghajanian GK (1977) Physiological evidence for habenula as major link between forebrain and midbrain raphe. Science 197:89-91.

Westerfield M (1995) The zebrafish book. Eugene, OR: University of Oregon.

Woods IG, Wilson C, Friedlander B, Chang P, Reyes DK, Nix R, Kelly PD, Chu F, Postlethwait JH, Talbot WS (2005) The zebrafish gene map defines ancestral vertebrate chromosomes. Genome Res 15:1307-1314.

Wullimann MF, Mueller T (2004) Teleostean and mammalian forebrains contrasted: evidence from genes to behavior. J Comp Neurol 475: 143-162.

Yañez J, Anadón R (1996) Afferent and efferent connections of the habenula in the rainbow trout (Oncorhynchus mykiss): an indocarbocyanine dye (DiI) study. J Comp Neurol 372:529-543.

Zirlinger M, Kreiman G, Anderson DJ (2001) Amygdala-enriched genes identified by microarray technology are restricted to specific amygdaloid subnuclei. Proc Natl Acad Sci U S A 98:5270-5275. 